the Auger rate can have a large effect on the fluorescence yield.

Aziz, Chergui and co-workers propose that the negative intensity observed in the Fe XAS spectrum is caused by the transfer of the excited electron from the iron atom to the surrounding water hydration shell (Fig. 1). This will cause the fluorescence yield to drop lower than the background signal, which is created by the surrounding water molecules that are also excited during the experiment. The transfer of the excited electron from the iron atom means that the number of electrons that can participate in the core-hole decay becomes smaller resulting in a lower fluorescence yield. For it to affect the fluorescence, however, it is essential that the electron transfer takes place before the core hole has a chance to decay. The lifetime of the hole in the iron core is of the order of a few femtoseconds, and therefore the electron transfer needs to occur on an even faster timescale. This means an ultrafast electron transfer that could occur within attoseconds.

What type of interactions can facilitate such a fast transfer of electrons to water? It has previously been seen by Näslund and co-workers ${ }^{3}$ that the XAS spectrum of the water molecules in the hydration shell of iron cations shows specific spectral features attributable to direct interactions between the molecular orbitals of the water and the $d$-electrons of the cation. Aziz, Chergui and co-workers show that the spectral features that have a lower fluorescence yield are of similar origin, but they probed this through the iron cation rather than the water molecules. The water-iron system thus forms a delocalized electronic state that will facilitate the ultrafast electron transfer to the hydration shell water on core excitation.

The study by Aziz, Chergui and coworkers leaves some questions open for future investigations. It is not yet clear how the balance between Auger and fluorescence decay rates will change with the number of electrons on the excited atom and how this would affect the fluorescence yield. Theoretical calculations of the decay rates could provide further insights. Another question is whether the electron transfer is only limited to the water molecules in the first hydration shell or if it can extend further into the liquid. The oxygen XAS study of this system by Näslund and co-workers ${ }^{3}$ indicated that the specific spectral features that involve mixing with Fe $d$-electrons are only related to the first hydration shell. This would suggest that the electron transfer could be localized to only the first shell. Although the electron transfer is shown by Aziz, Chergui and co-workers to occur on a timescale faster than the core-hole lifetime, this is only an upper bound and it would be most interesting if the timescale of this fundamental process could be determined more precisely with ultrafast techniques. In this respect, new free-electron X-ray laser facilities could perhaps be used to provide sub-femtosecond timescale resolution of these fascinating core excitation-induced electron-transfer processes.

Anders Nilsson is in the Photon Science Department of the SLAC National Accelerator Laboratory, 2575 Sand Hill Road, MS 69, California 94025 Menlo Park, USA.

e-mail:nilsson@slac.stanford.edu

References

1. Ball, P. Chem. Rev. 108, 74-108 (2008)

2. Marcus, Y. Chem. Rev. 109, 1346-1370 (2009).

3. Näslund, L. Å. et al. J. Phys. Chem. A 107, 6869-6876 (2003)

4. Aziz, E. F., Rittmann-Frank, M. H., Lange, K. M.,

Bonhommeau, S. \& Chergui, M. Nature Chem.

2, 853-857 (2010).

5. Stöhr, J. NEXAFS Spectroscopy (Springer, 1992)

Published Online: 8 August 2010

\title{
MICROSCOPY
}

\section{Imaging interactions}

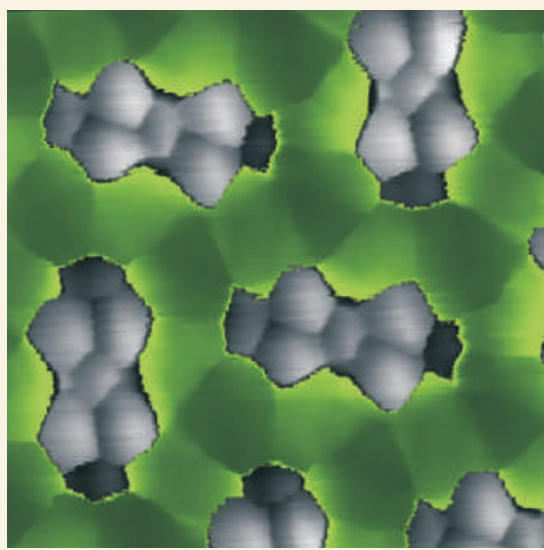

Determining the arrangement of the atomic constituents of molecules is vital to many aspects of chemistry and has been mostly solved by various advances in spectroscopy and crystallography. Recent developments have made it possible for such analyses to be more direct: for example, atomic force microscopy has been used to image the bonding within molecules. Uncovering

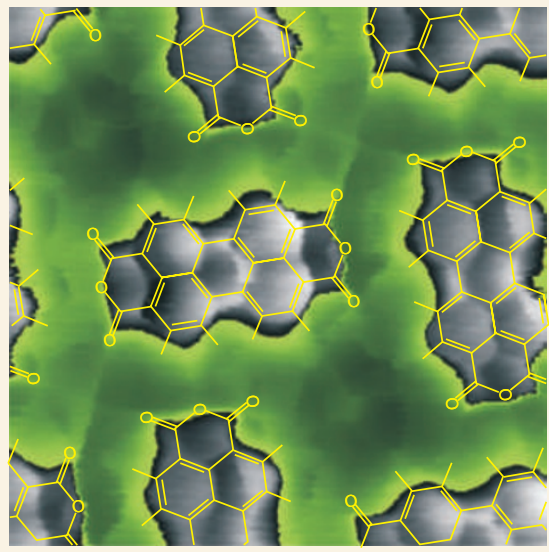

how different molecules interact with one another, however, is more problematic, and proving the presence of such non-covalent bonding has been restricted to indirect inference. Now Stefan Tautz and colleagues at Forschungszentrum Jülich in Germany, have shown that intermolecular bonds can be directly imaged using scanning tunnelling hydrogen microscopy (STHM), a variant of
STM whereby the probe tip is sensitized with molecular hydrogen (J. Am. Chem. Soc. 132, 11864-11865; 2010).

They studied the behaviour of 3,4,9,10-perylenetetracarboxylic-dianhydride (PTCDA) on a gold surface first using conventional STM, which shows how the molecules are arranged, and then using STHM. The STHM images (pictured) gave more detail than the conventional images, revealing not only various features of the molecular structure, but also a pattern of lines between the molecules. These originate at oxygen and hydrogen atoms and align well with expected intermolecular interactions. The physical basis for why this technique is able to produce such clear images of intermolecular interactions is, however, yet to be understood.

\section{GAVIN ARMSTRONG}

The original version of this story first appeared on the Research Highlights section of the Nature Chemistry website. 